\title{
Micromechanics-based model for trends in toughness of ductile metals
}

\author{
T. Pardoen ${ }^{\mathrm{a}, *}$, J.W. Hutchinson ${ }^{\mathrm{b}}$ \\ a Département des Sciences des Matériaux et des Procédés, PCIM, Université Catholique de Louvain, Place Sainte Barbe 2, \\ B-1348 Louvain-la-Neuve, Belgium \\ ${ }^{\mathrm{b}}$ Division of Engineering and Applied Sciences, Harvard University, 02138 Cambridge, MA, USA
}

Received 7 June 2002; received in revised form 17 July 2002; accepted 17 July 2002

\begin{abstract}
Relations between fracture toughness and microstructural details have been calculated for ductile materials based on a dilatational plasticity constitutive model that has recently been proposed. The model generalizes the Gurson model to account for both void growth and coalescence with explicit dependence on void shape and distribution effects. Based on a small scale yielding formulation of crack growth, toughness trends are determined as a function of yield stress, strain-hardening, initial porosity, void shape and spacing as well as void spacing anisotropy. Distinctions are drawn between the engineering fracture toughness, which is typically associated with $0.2 \mathrm{~mm}$ of crack growth, and the theoretical toughness based on coalescence of the crack tip with the first void ahead of it. Comparison with one set of experimental data for a steel is made for which a fairly complete characterization of the microstructure is available.
\end{abstract}

(C) 2002 Acta Materialia Inc. Published by Elsevier Science Ltd. All rights reserved.

Keywords: Metals; Fracture toughness; Ductile; Theory and modeling; Voids

\section{Introduction}

In the context of material science, the fracture toughness, at cracking initiation is usually taken as the relevant parameter for indexing the cracking resistance of materials. The $J$ integral at cracking initiation, $J_{\text {Ic }}$, is employed to characterize the toughness of ductile alloys that exhibit significant amount of crack tip plasticity. In general, $J$ is mea-

\footnotetext{
* Corresponding author.

E-mail addresses: pardoen@pcim.ucl.ac.be (T. Pardoen); hutchinson@husm.harvard.edu (J.W. Hutchinson).
}

sured experimentally as a function of the crack extension giving the so-called ' $J_{\mathrm{R}}$-curve' of the material. The full $J_{\mathrm{R}}$-curve obtained on laboratory specimens provides three important quantities [1]: (1) the initiation toughness $J_{\text {Ic }}$, defined in an engineering sense, i.e. at a predetermined amount of crack growth, or, if it can be detected, at the physical event of cracking initiation; (2) an average tearing resistance $\mathrm{d} J / \mathrm{d} a$; and (3) when sufficient crack growth is allowed and sufficiently large specimens are used, the steady state fracture toughness, $J_{\mathrm{ss}}$. Engineering analysis of structural integrity requires both initiation and crack growth data, at least for the early stages of the $J_{\mathrm{R}}$-curve. The emphasis in 
this paper is on the material science view of toughness by focusing on the crack initiation toughness of ductile metal alloys. The failure mechanism is void growth and coalescence, and the effort here is to relate fracture toughness to details of the microstructure such as void volume fraction, void shape and void distribution.

Fig. 1 depicts the model envisioned for addressing the cracking in ductile metal failing by the void growth mechanism. The initial geometry is a precrack of opening $\delta_{0}$ in an idealized material having regularly distributed voids with initial spacing $X_{0}$ and radius $R_{0}$ in the crack plane. The crack is long, and small scale yielding is assumed to apply. The material is elastic-plastic characterized by the ratio of the normalized yield stress, $\sigma_{0} / E$, Poisson's ratio, $v$, and a strain-hardening exponent $n$. The dimensionless geometrical microstructural parameters are $\chi_{0}=X_{0} / R_{0}, W_{0}=R_{\mathrm{zo}} / R_{0}$, and $\chi_{0}=$ $Z_{0} / X_{0}$. The initial void porosity $f_{0}$ can be expressed in terms of these last three variables.

In general, the problem of Fig. 1 requires a full numerical analysis of crack tip blunting coupled to a fracture process zone model. There are three main types of analyses that have been proposed in the literature to predict fracture toughness based on the model of Fig. 1.

- Finite element calculations are performed with the voids explicitly modeled by a refined FE mesh [2-9]. These analyses accurately model

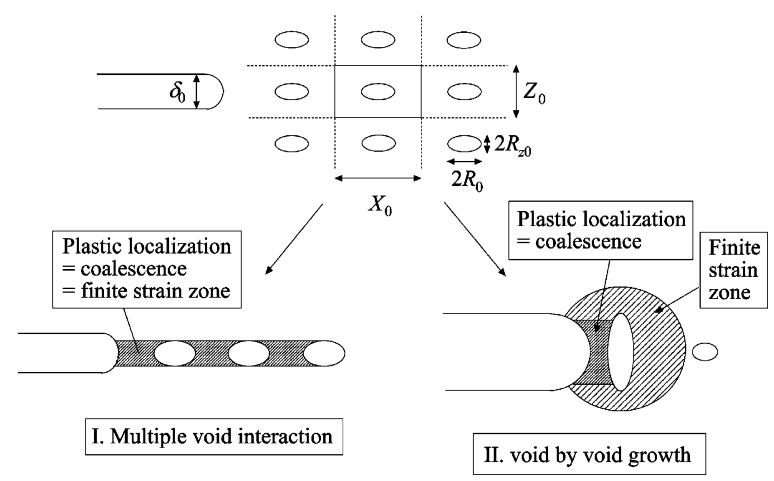

Fig. 1. The initial geometry of a precrack in a ideal material with regularly distributed spheroidal inclusions; sketch of the two ideal mode of crack initiation, i.e. multiple void interaction and void by void process. the growth and coalescence process while properly accounting for the length scale introduced by the void spacing. This approach still requires a criterion for the final failure of the intervoid ligament in order to simulate crack propagation (e.g. by modeling shear localization within the ligament due to a second population of smaller voids). This approach is computationally intensive and is able to account for only a few voids ahead of the crack tip.

- An alternative approach, which has been pursued mainly by groups in France, Germany, the UK and the US [10-21], employs a constitutive model, such as the Gurson model, that accounts for the damaging effect of voids. The constitutive model is implemented in a finite element code to simulate the initiation and growth of the crack. The approach requires the introduction of a length scale in the model related to the spacing between voids. This is usually accomplished by tying the element size to the void spacing, calibrated to crack growth resistance data.

- A third type of analysis makes use of cohesive zone surfaces to model the fracture process in ductile metals [22-25]. This approach is limited to materials whose void volume fraction is sufficiently large such that the fracture process involves multiple voids growing and coalescing on a plane ahead of the tip. A characteristic length is introduced since the cohesive properties involve the work of separation and the cohesive stress.

The second and third approaches have been applied with considerable success to analyze fracture integrity of structural components under large scale yielding conditions. Three dimensional (3D) aspects of crack initiation and growth have also been simulated $[18,21,25]$. The success of this approach is underpinned by the requirement that the microstructural parameters (the void volume fraction, void spacing, etc.) must be set such that the model reproduces experimental crack data for specific specimens. Once calibrated, these approaches have accurately accounted for a wide range of constraint effects. Thus, the methods have been used to study crack initiation and growth for specific materials, but they have not been used as 
a tool for material sciences to explore trends in toughness as dependent on microstructural parameters. This is the objective of the present paper.

In this paper we use the small-scale yielding, plane strain model of Fig. 1 to compute trends in fracture toughness. Three new aspects are introduced in the constitutive model that have not been considered in previous modeling efforts devoted to linking fracture toughness to the microstructure [23,26-28]:

- Non-spherical voids ranging from penny-shape cracks to highly prolate voids, that are common in industrial metal alloys have been accounted for. These shapes result from the metal forming process during which globular second phases experience severe deformations. Generally, forming processes also bring about a preferential orientation for the principal axis of the second phases and induce some degree of anisotropy in the void distribution. Furthermore, initially spherical void tends to become prolate at low stress triaxiality and oblate at high stress triaxiality.

- A physical void coalescence mechanisms will be used for modeling the coalescence between voids and between the first void and the crack.

- One of the most important ingredients of the void coalescence model is the relative spacing between the voids. Its role will thus be studied.

The inclusion of the three points discussed before is made possible by a recent extension of Gurson's model [29] of plastic solids containing voids to include shape, coalescence and distribution effects [30] based on contributions by Gologanu et al. [31] and Thomason [32].

The plan of the paper is as follows. An outline of the constitutive relation and the finite element computational model is presented in Section 2. This is followed in Section 3 by a critical assessment of the model based on comparisons with recent results obtained for a detailed calculation that provides a high resolution of both the crack tip and the voids. Section 4 synthesizes the main results of the study revealing the dependence on the influential microstructural parameters in the form
$\frac{J_{\mathrm{Ic}}}{\sigma_{0} X_{0}}=F\left(\frac{\sigma_{0}}{E}, n, f_{0}, W_{0}, \lambda_{0}\right)$

The effect of the initial porosity, shape and relative spacing on the toughness will be discussed. Section 4 will also incorporate results dealing with the difference between the engineering toughness defined after a predetermined amount of crack growth and the physical toughness at the 'true' cracking initiation. The model captures the variations of the fracture toughness as a function of the orientation of the crack plane relative to a distribution of voids. The model will be applied in Section 5 to analyze the results obtained by Bauvineau [33] on a rolled steel as an illustration of this orientation dependence and a preliminary experimental assessment. The paper ends with suggestions for future investigation.

\section{Void growth model and computational methods}

\subsection{An enhanced model for void growth and coalescence}

The extended Gurson model used in this paper, to account for the effect of the growth and coalescence of voids on the behavior of the material has been presented in details in Ref. [30]. It is based on the works by Gologanu et al. [31] for the account of the void aspect ratio and by Thomason [32] for the onset of coalescence with extensions to strain-hardening and for the modeling of the coalescence process [34]. A summary of the structure of the model is given in Appendix A. The model is based on two different solutions for the expansion of a void in an elastoplastic material: one solution is called 'void growth' corresponding to diffuse plasticity around the void, and the other is called 'void coalescence' corresponding to localized plasticity in the intervoid ligament. These two solutions can be presented in the form of two distinct plastic yield surfaces supplemented by evolution laws for the internal variables of the model (the porosity $f$, the void aspect ratio $W$, the relative void spacing, $\chi$ and the mean yield stress of the matrix material $\sigma_{\mathrm{y}}$ ) and the normality rule 
for the plastic strain increment. These two yield surfaces are

$$
\begin{gathered}
\Phi_{\text {growth }} \equiv \frac{C}{\sigma_{\mathrm{y}}^{2}}\left\|\boldsymbol{\Sigma}^{\prime}+\eta \boldsymbol{\Sigma}_{\mathrm{h}} \boldsymbol{X}\right\|^{2}+2 q(g+1)(g \\
+f) \cosh \left(\kappa \frac{\Sigma_{\mathrm{h}}}{\sigma_{\mathrm{y}}}\right)-(g+1)^{2}-q^{2}(g+f)^{2}=0
\end{gathered}
$$

and

$\Phi_{\text {coalescence }} \equiv \frac{\Sigma_{\mathrm{e}}}{\sigma_{\mathrm{y}}}+\frac{3\left|\Sigma_{\mathrm{h}}\right|}{2 \sigma_{\mathrm{y}}}-F(W, \chi)=0$

(see Appendix A for the definition of the parameters entering these formulas). The first yield surface $\Phi_{\text {growth }}$ is a Gurson-type yield surface derived by Gologanu-Leblond-Devaux model and extended to strain-hardening materials in Ref. [30] (see Appendix A). The yield function $\Phi_{\text {coalescence }}$ has been developed in the spirit of the work by Thomason, extended to the full coalescence response and to strain-hardening. The following uniaxial response has been chosen for this study:

$$
\begin{aligned}
& \frac{\sigma}{\sigma_{0}}=\frac{E \varepsilon}{\sigma_{0}} \text { when } \sigma<\sigma_{0}, \\
& \frac{\sigma}{\sigma_{0}}=\left(1+\frac{E \varepsilon^{\mathrm{p}}}{\sigma_{0}}\right)^{n} \text { when } \sigma>\sigma_{0} .
\end{aligned}
$$

Fig. 2 illustrates the competition between the two modes of plastic deformation. The yield surfaces and loading history correspond to a high constraint situation (a constant stress triaxiality of 3) relevant to a material element in front of a crack tip. The material is characterized by $\sigma_{0} / E=$ $0.002, n=0.1, f_{0}=0.01, W_{0}=1$ and $\lambda_{0}=1$. Fig. 2a corresponds to elastic loading: the stress state lies within the elastic domain of the two yield surfaces. As shown in Fig. 2b, the first yield surface to be reached is $\Phi_{\text {growth. }}$. In the beginning of the deformation, the voids are small and the spacing is relatively large resulting in a diffuse plastic deformation. With increasing deformation $\Phi_{\text {growth }}$ first tends to expand due to hardening and then to contract due to void growth softening. Void growth and ligament reduction also induces a contraction of $\Phi_{\text {coalescence. }}$ Fig. $2 c$ corresponds to an overall strain just before the onset of coalescence and Fig. $2 \mathrm{~d}$ to an overall strain just after the onset of coalescence. When the two yield surfaces intersect at the current loading point, the transition to coalescence occurs. With increasing deformation, the coalescence yield surface tends to contract very rapidly towards the zero stress state. Fig. 2e shows the two yield surfaces far into the coalescence stage.

For almost all loading histories of interest, coalescence when initiated will continue until complete failure. In some instances, when abrupt changes of loading direction occur, it may happen that the system returns to a void growth mode of deformation. This case is not allowed in the current implementation of the model (see next subsection). The unlikely event wherein coalescence is interrupted with the resumption of diffuse void growth is not expected for the present work on mode I cracking. The stresses at a material point may evolve non-proportionaly, but they will not undergo abrupt changes in loading direction. Note that finally, as it stands, the model does not account for other mechanisms that might intervene to produce the final rupture of the ligament, such as local cleavage or localization due to a second population of smaller voids. This point will also be addressed in Section 3.

\subsection{Plane strain, small scale yielding}

The constitutive model described in the previous section and in Appendix A has been implemented in the general purpose finite element code 'ABAQUS Standard' through a User defined MATerial subroutine (UMAT) [35] with a fully implicit integration scheme based on a classical return mapping algorithm [36]. The set of 10 first order differential equations involved in the void growth model are solved using the Newton-Raphson algorithm. The Aravas scheme [37] has been applied for the integration of the coalescence model permitting a significant reduction in the size of the system of differential equations to be solved at each integration station during the return to the yield surface.

A small scale yielding formulation of the crack problem has been adopted. As indicated earlier, the objective is the prediction of the relationship between fracture toughness and the microstructure 
(a)

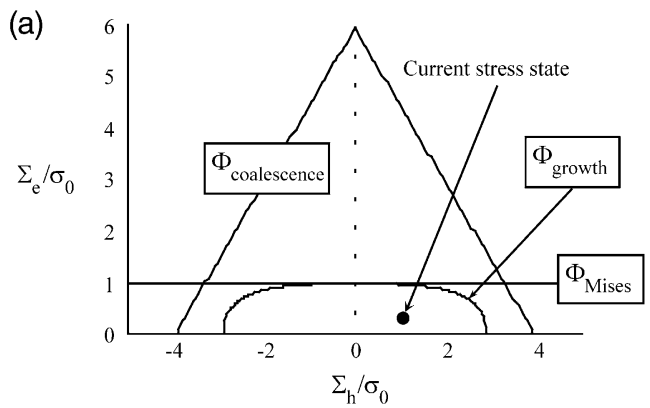

(b)

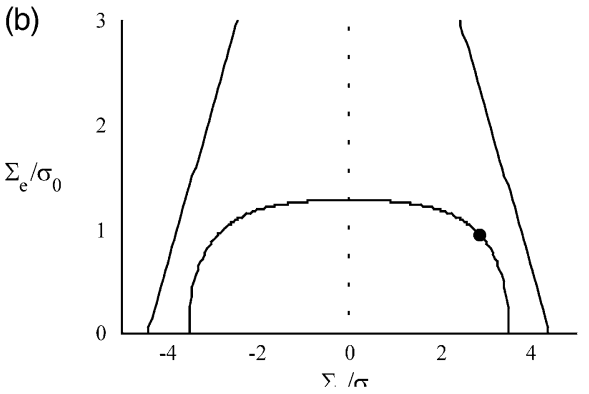

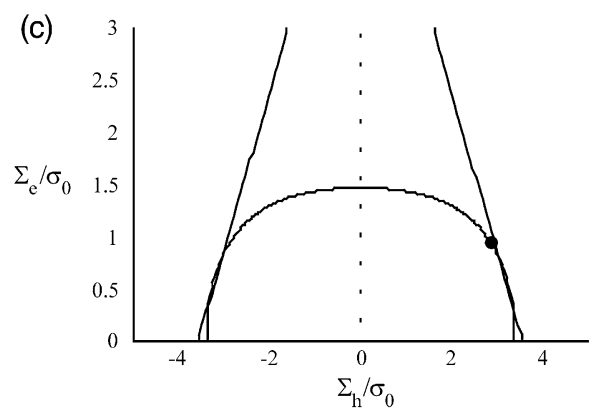

(d)

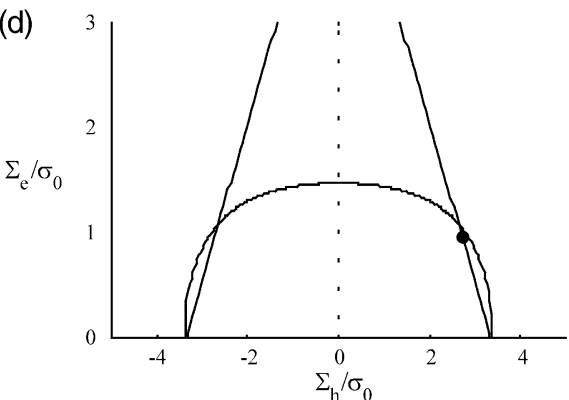

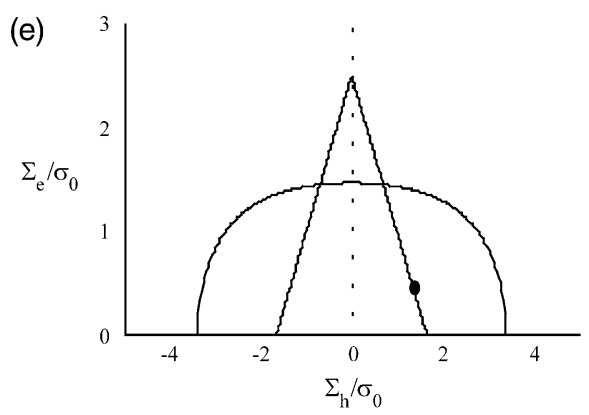

Fig. 2. Transition from (a) elastic behavior, to (b) and (c) plastic void growth, to (d) and (e) plastic void coalescence in terms of the variation of the yield surfaces and current loading point as a function of increasing straining. Calculations performed for a constant stress triaxiality equal to 3 .

in metal alloys. An infinite cracked solid is modeled using a semi-circular finite element mesh whose radius $R$ is $10^{7}$ times larger than the size of the representative element size in the fracture process region (Fig. 3). A large radius is necessary to ensure small scale yielding in the calculations performed on high toughness materials with low porosity and high strain-hardening such that the plastic zone size remains smaller than about $1 \%$ of $R$. Displacement increments corresponding to the mode I plane strain $K$-field were prescribed on the outer boundary while the crack surface is traction free. A regular mesh with constant element size suitable for modeling of crack advances was designed in the region in front of the crack tip. A finite strain set-up is used. The crack tip is a round notch of diameter $\delta_{0}$. In this paper, results will presented only for $\delta_{0} / X_{0}=0.1$ (i.e. a very sharp initial crack characteristic of a fatigue precrack.) To contain the computational time, only a few rows of elements ahead of the tip were modeled with damage. The rest were modeled using $J_{2}$ flow theory. 
(a)

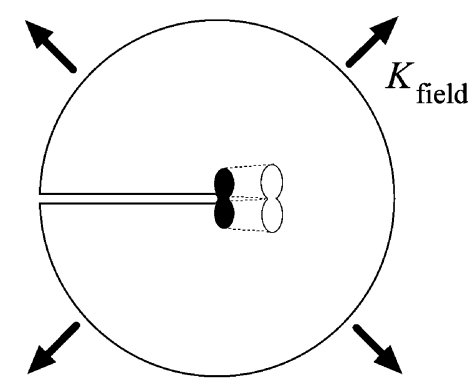

(b)

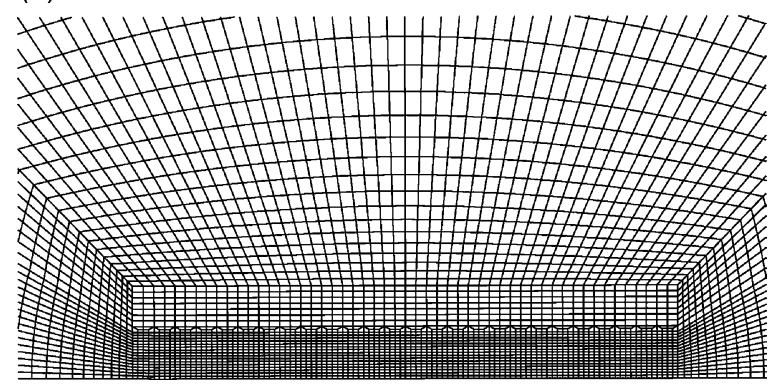

Fig. 3. (a) Description of the small scale yielding formulation; and (b) zoom of the finite element mesh in the near crack tip region.

\subsection{The fracture length scale}

As is evident from (1), the void spacing, $X_{0}$, is the length scale setting the fracture toughness. The constitutive law derived from the potentials (2) and (3) can be regarded either as an average over many voids or as the relation governing a single void in a material element. Indeed, the original derivation of Gurson was based on a single void in a spherical element. In the latter view, which is adopted here, the size of the finite elements is directly related to void spacing in the portion of the mesh that experiences void growth and coalescence, i.e. in the fracture process zone. This is the approach that has been adopted in most ductile fracture simulations based on the Gurson model or its derivatives. As mentioned in the Section 1, a critical step in the calibration process of such models to experimental data involves the adjustment of $X_{0}$. In all cases here, the elements in the fracture process zone are taken to be square and of dimension $X_{0}$. Thus, normalization of the toughness by the only length scale $X_{0}$ obviously leads to results that are independent of the degree of mesh refinement.

\section{Assessment of the model}

In a recent work, Tvergaard and Hutchinson [9] carry out plane strain, small-scale yielding computations of mode I fracture initiation and tearing in a $J_{2}$ elastic-plastic solid containing a single row of cylindrical voids. Deformation around the voids was fully resolved using the mesh as shown in Fig. 4. A fracture criterion is postulated for the final failure of the ligaments between voids, as otherwise calculations would have to be interrupted because of severe mesh distortion. All force carried by a ligament is terminated when its width is reduced to the reduction factor, $R$, times the initial ligament length. Typically, $R$ was chosen as $1 / 2$ or $1 / 3$. The study in Ref. [9] offers an opportunity to critically assess the model proposed in this paper. For that purpose, the failure criterion based on the reduction of ligament length was also implemented in our model. Identical parameters to those in Ref. [9] for the flow properties and initial microstructure have been used: $\sigma_{0} / E=0.003, n=0.1$, $W_{0}=1$ and $\lambda_{0}=1$.

Fig. 5 compares the results for $R=1 / 2$ and $1 / 3$. The variations of $J_{\mathrm{Ic}} / \sigma_{0} X_{0}$ as a function of $f_{0}$ for the two models are very similar. The predictions of the present model are slightly above those of Ref. [9]. Some discrepancy of course arises from the approximation inherent in the present approach. In addition, some discrepancy is also expected from the fact that the voids in the present model are $3 \mathrm{D}$ and initially spherical, while those in the more refined simulation are two-dimensional (2D) and initially circular cylindrical. Under otherwise similar situations, spherical voids undergo delayed coalescence relative to cylindrical voids [38]. In any case, the close correspondence between the two sets of results lends confidence in the present model.

The good agreement between the two models also means that the present model correctly captures the transition of failure mode described in Ref. [9]: at sufficiently high porosity, the void near the tip is influenced by its nearest neighbor, which 


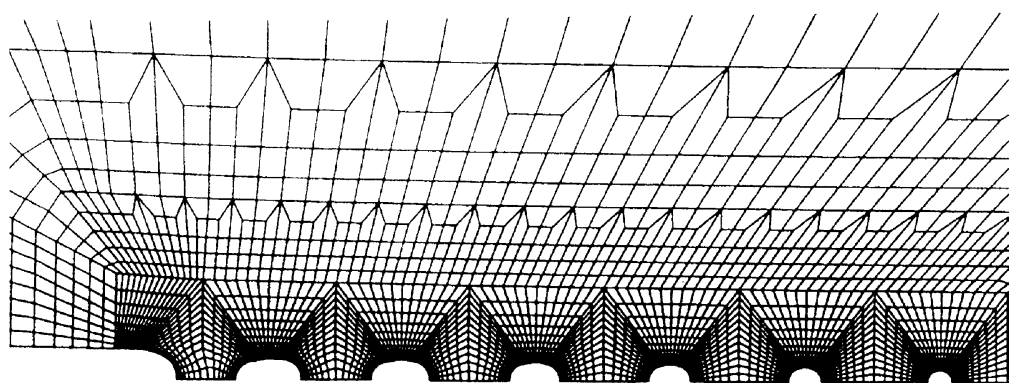

Fig. 4. Finite element 2D small scale yielding simulations of ductile crack growth with discrete voids [9].

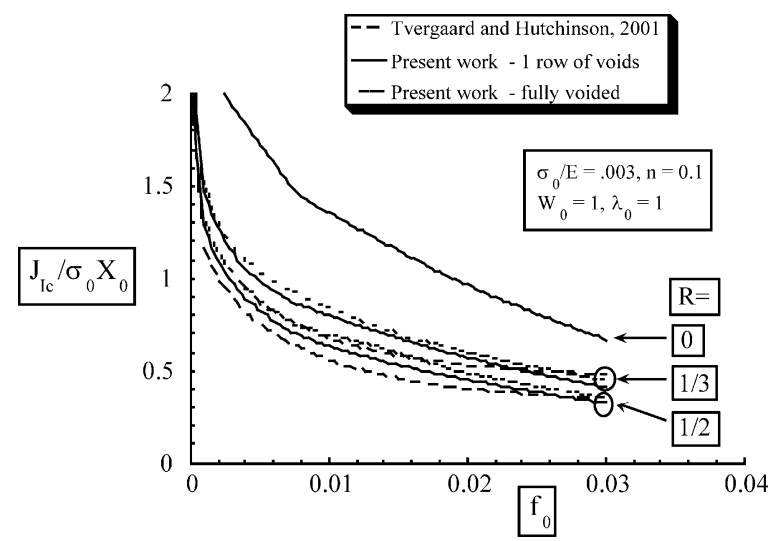

Fig. 5. Variation of the fracture toughness as a function of the initial porosity for different values of the final failure criterion (different $R$ ). Comparison with the results obtained by Tvergaard and Hutchinson [9].

experiences almost the same rate of growth. The interaction among the voids, including voids even farther from the tip, results in significantly higher rate of void growth for all of the voids. Coalescence between several voids and with the crack starts early, almost simultaneously. This is the multiple void interaction mechanism. For sufficiently small void volume fraction, a single void process prevails, which is essentially the process envisioned by the Rice-Johnson [26] model. The void near the tip grows with little influence from its nearest neighbor further from the tip. The increase of the normalized toughness with decreasing porosity is more marked in this regime. This is the void by void growth mechanism. In order to more quantitatively detect the change of failure mode,
Fig. 6 displays the variation of the size of the fracture process zone at initiation as a function of the initial porosity. The size of the fracture process zone is given by the number of elements which are within the coalescence stage. The transition takes place between $f_{0}=10^{-3}$ and $10^{-2}$, depending on the value of $R$ chosen for the final failure process. For materials with significant crack growth resistance, the fracture process zone length increases as the crack advances with consequence that materials exhibiting void by void growth at initiation may transition to the multiple void interaction mechanism as growth proceeds. Thus, the transition from one mechanism to another effectively takes place at a somewhat lower porosity than Fig. 6 would suggest.

Two sets of results have also been plotted in Fig. 5 for computations performed: (1) a single row of void elements ahead of the tip; and (2) void

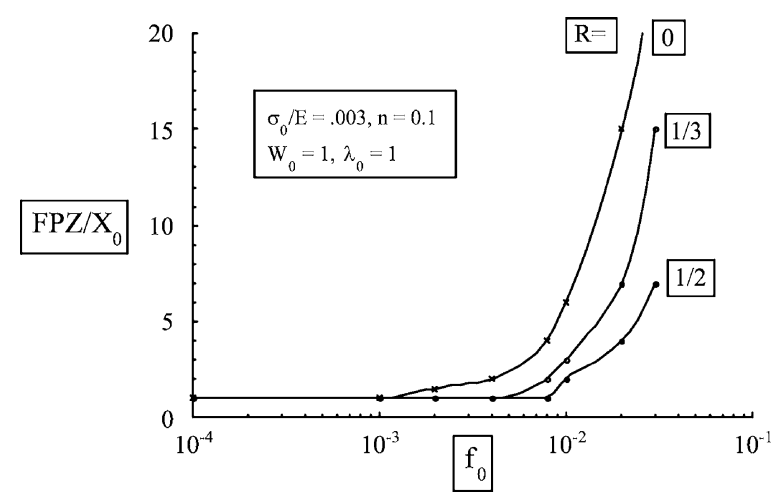

Fig. 6. Variation of the fracture process zone size as a function of the initial porosity for different values of the final failure criterion (different $R$ ). 
elements throughout the entire material. Otherwise, the parameters of the two cases are identical. There is little difference between two sets of results, although the fully voided material is predicted to be slightly tougher than that with a single row of voids. In each case, growth and coalescence is confined to the plane of voids ahead of the tip. In the fully voided material, voids on both sides of the fracture plane tend to relax the stresses on the voids on the fracture plane, thereby delaying their growth and localization. Note that the difference between a material containing a single row of voids and a fully voided material becomes much more important during crack advance: the model then predicts tearing moduli that are significantly different.

Fig. 5 also shows results for $R=0$, i.e. failure by reducing the ligament width to 0 by ductile stretching. The effect of $R$ is important, and the appropriate choice of $R$ is material dependent. For instance, in most steels, a second population of smaller voids or shear localization (in case of zigzag cracking in some very high strength alloys [39]) lead to premature ligament failure; then values of $R$ equal or larger than $1 / 3$ may be realistic. However, in other materials, such as copper [40] voids grow until final impingement with the crack. For the remaining of the paper, all results presented will be computed with $R=0$. The results will thus, provide an upper bound for the toughness of materials having some degree of ligament embrittlement. Finally, it is interesting to note that average stretching strain in the first crack tip element is between 0.1 and 0.2 at failure, when $R=1 / 2$ and between 0.5 and 1 when $R=0.05$. Experimental data on HSLA steels [41], show, from local strain measurement, strains of about 0.5-0.75 in front of the crack tip at cracking initiation. More observations of this kind would be valuable in helping predictive micromechanical models for ductile fracture.

\section{Fracture toughness trends}

In addition to initial porosity and void spacing, that have been discussed before, this section will explore the effect on toughness of normalized yield

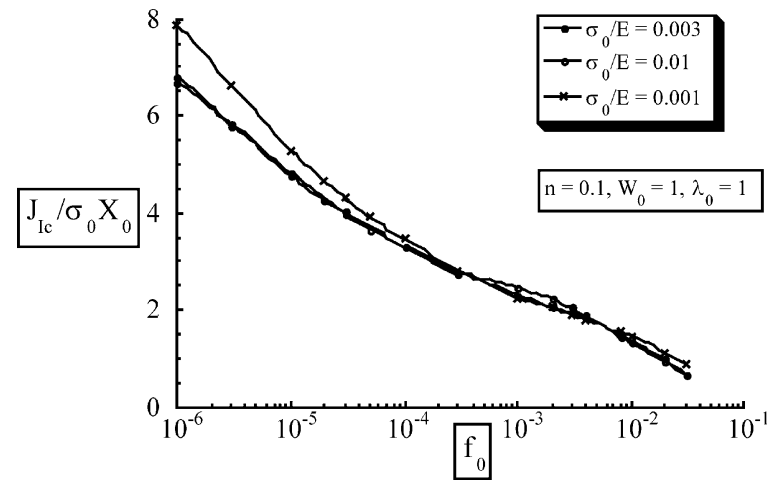

Fig. 7. Effect of the ratio $\sigma_{0} / E$ on the fracture toughness for different initial porosity.

stress, $\sigma_{0} / E$, strain-hardening, $n$, void aspect ratio, $W_{0}$, and void spacing distribution as measured by $\lambda_{0}$. The section ends with a discussion of the role of crack growth resistance on the definition of fracture toughness.

\subsection{Dependence on flow properties}

Fig. 7 shows the effect of the ratio $\sigma_{0} / E$ on $J_{\text {Ic }} / \sigma_{0} X_{0}$ for $n=0.1$. As expected from previous results [9], fracture toughness scales linearly with $\sigma_{0}$, but is otherwise essentially independent of $\sigma_{0} / E$. Fig. 8 exhibits the effect of the strain-hardening index, $n$, on the fracture toughness for a material with $\sigma_{0} / E=0.003, v=0.3, W_{0}=1$, and $\lambda_{0}=1$. Strain hardening has a major influence on fracture toughness.

The fact that the fracture toughness is linearly

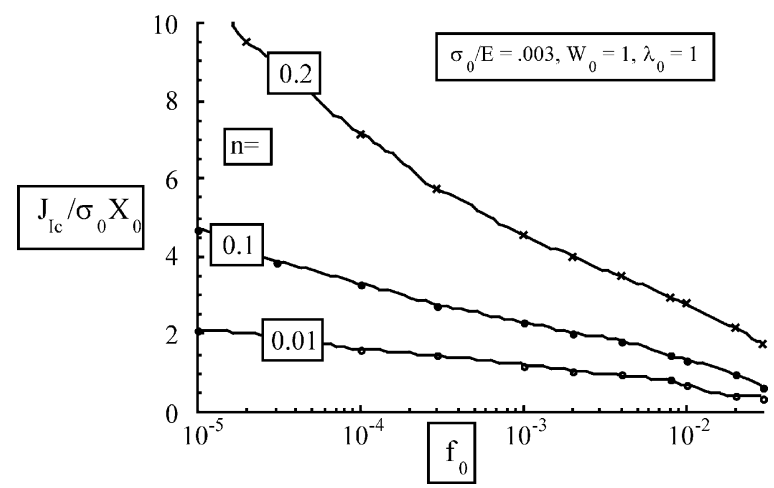

Fig. 8. Effect of the strain-hardening exponent $n$ on the fracture toughness for different initial porosity. 
proportional to the yield stress (Fig. 7) seems to contradict much experimental evidences showing that the toughness $\left(J_{\text {Ic }}\right)$ of a family of alloys usually decreases with increasing yield stress $\left(\sigma_{0}\right)$. Several points are relevant to this apparent contradiction.

First, in many alloys, an increase of $\sigma_{0}$ by metallurgical intervention is usually accompanied by a decrease of the strain-hardening index $n$ that has the opposite effect on the toughness. For instance, these countervailing trends occur in precipitation hardening of aluminum alloys, where the precipitate do not, in general, take part to the failure process. An example can be found in a recent study by Dumont et al. $[42,43]$ on the fracture toughness of 7000 Al alloys: an under-aged Al7040 characterized by $\sigma_{0}=385 \mathrm{MPa}, n \approx 0.2$ is compared to the over-aged alloy characterized by $\sigma_{0}=460 \mathrm{MPa}$ $n \approx 0.1$ Both materials primarily fail by a ductile void growth mechanism (which is not true for the peak-aged alloy showing intergranular fracture). The initial void volume fraction is identified with the volume fraction of intermetallic particles $f_{0}=$ $5 \times 10^{-3}$ (intermetallic particles are not affected by ageing treatment). The ratio of the two fracture toughnesses was measured as $J_{\mathrm{c}}^{\text {over-aged }} / J_{\mathrm{c}}^{\text {under-aged }} \approx 0.55$, in good agreement with the ratio that can be extracted from Fig. 8: for $f_{0}=5 \times 10^{-3}$ and $n=0.1, J_{\text {Ic }} / \sigma_{0} X_{0}=1.72$ and for $f_{0}=5 \times 10^{-3}$ and $n=0.2, J_{\mathrm{Ic}} / \sigma_{0} X_{0}=3.3$; thus, the model predicts a ratio $J_{\mathrm{c}}^{\text {over-aged }} / J_{\mathrm{c}}^{\text {under-aged }}=172 \sigma_{0}^{\text {over-aged }} / 3.3 \sigma_{0}^{\text {under-aged }}=$ 0.62 , assuming that the damage sites have not been affected by the ageing (i.e. $X_{0}$ is constant).

Secondly, the present model does not incorporate a void or microcrack nucleation criterion. In many instances, an increasing yield strength will affect the nucleation stage by raising the stress on the second phase particles or grain-boundaries. A larger yield stress may also favor nucleation on smaller particles or on a second population of particles at an earlier stage of the deformation.

However, if all other parameters, including strain-hardening, can be kept constant, higher yield stress directly implies higher fracture toughness. A good illustrative example is given by the decrease with increasing temperature of the fracture toughness of ferritic steel in the upper shelf region. For the typical temperature range covered when measuring a ductile-brittle fracture transition curve, no modification of microstructure or hardening is expected. The only effect is a decrease of yield stress with increasing temperature.

\subsection{Dependence on void aspect ratio}

Fig. 9 presents the variation of $J_{\mathrm{Ic}} / \sigma_{0} X_{0}$ as a function of $f_{0}$ for different initial void shapes ranging from $W_{0}=1 / 10$ (highly oblate) to $W_{0}=10$ (highly prolate), for $\sigma_{0} / E=0.001, n=0.1$, and $\lambda_{0}=1$. The effect of the initial void shape is in accord with intuition. At a given porosity, prolate voids have a smaller area fraction projected onto the fracture plane than spheres and conversely for oblate shapes. Thus, prolate shapes increase $J_{\text {Ic }} / \sigma_{0} X_{0}$ while oblate shapes reduce it relative to spherical voids at the same volume fraction. Prolateness tends to delay void coalescence by affecting the constraint in the ligament between the voids. Moreover, prolate voids imply a larger void spacing for a given porosity. The right end of each curve for the prolate voids in Fig. 9 corresponds to the limit porosity at which the void touches the upper and lower boundaries of the cell. For oblate voids, the limiting porosity where $J_{\mathrm{Ic}} / \sigma_{0} X_{0}=0$ corresponds to contact of the void with the lateral sides of the cell, reducing the strength of the material to 0 in the direction perpendicular to the fracture plane. These two limiting porosity values are readily found to be:

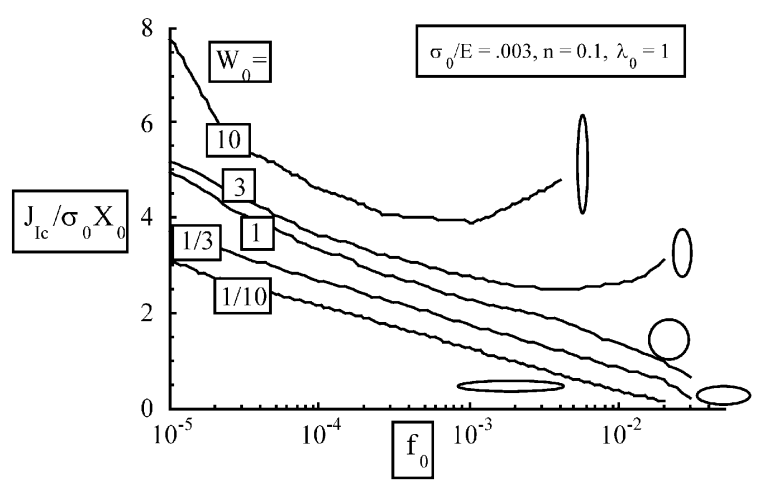

Fig. 9. Effect of the initial void shape on the fracture toughness for different initial porosity. 
$\left(f_{0}\right)_{\text {prolate }}=\frac{2}{3} \frac{\lambda_{0}^{2}}{W_{0}^{2}}$ and $\left(f_{0}\right)_{\text {oblate }}=\frac{2}{3} \frac{W_{0}}{\lambda_{0}}$.

The results of Fig. 9 can be used to guide understanding of, as well as to predict the variation of, the fracture toughness as a function of the loading direction for rolled plates with preferential orientation of the second phase. An application to a material with anisotropic fracture toughness is discussed in Section 5. Clearly, void shape has a significant effect on fracture toughness. It is one of the main reasons for fracture anisotropy in ductile failure.

\subsection{Dependence on relative void spacing}

Fig. 10 shows sketches of the crack tip region in two specimens, made of a material with spherical voids that are distributed non-uniformly in the sense that the spacing in one direction is different from that in the other. One can imagine these two specimens to have been machined from the same material with their crack planes oriented at $90^{\circ}$ to one another. Specifically, a representative cell in the process zone of Specimen $\mathrm{A}$ has $X_{0}^{\mathrm{A}}=a Z_{0}^{\mathrm{A}}$ with $\lambda_{0}^{\mathrm{A}}=Z_{0}^{\mathrm{A}} / X_{0}^{\mathrm{A}}=1 / a$, while Specimen $\mathrm{B}$ $\left(X_{0}^{\mathrm{B}} \equiv Z_{0}^{\mathrm{A}}, Z_{0}^{\mathrm{B}} \equiv X_{0}^{\mathrm{A}}\right)$ has $Z_{0}^{\mathrm{B}}=a X_{0}^{\mathrm{B}}$ with $\lambda_{0}^{\mathrm{B}}=a$. A

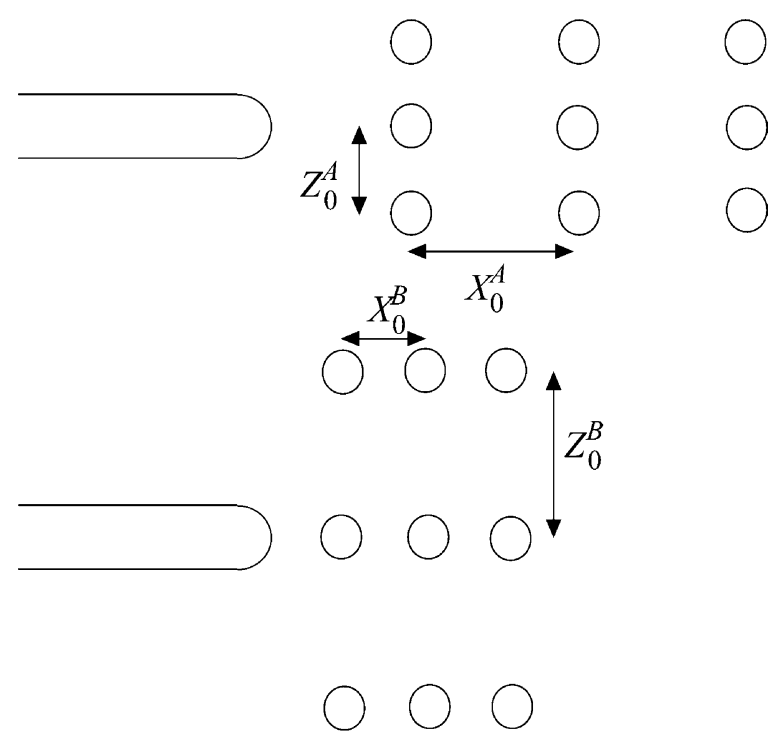

Fig. 10. Two different crack plane orientations in a material exhibiting initially anisotropic void spacing. similar distribution of weakly bonded inclusions (typically with $a$ lower than 2) is not uncommon in industrial alloys as a result of the forming process. The question to be addressed in this section is: What is the difference in fracture toughness of the two specimens resulting from this anisotropic void distribution?

As the initial porosity is the same in both Specimens $\mathrm{A}$ and $\mathrm{B}$, the results of the last sections could be applied if it were assumed that a change in the anisotropy of void spacing, $\lambda_{0}=Z_{0} / X_{0}$, does not, to the first order, affect $J_{\text {Ic }} / \sigma_{0} X_{0}$. Then, the difference in the two specimens is fully accounted for by the fact that $X_{0}^{\mathrm{A}}=a X_{0}^{\mathrm{B}}$, from which it follows that Specimen A is tougher than Specimen B by the factor $J_{\mathrm{Ic}}^{\mathrm{A}} / J_{\mathrm{Ic}}^{\mathrm{B}}=a$. However, $\lambda_{0}$ does influence $J_{\text {Ic }} / \sigma_{0} X_{0}$ as can be seen in Fig. 11 where the computed result is compared with the 'simplistic' estimate $J_{\mathrm{Ic}}^{\mathrm{A}} / J_{\mathrm{Ic}}^{\mathrm{B}}=a$. It is important to note that the void growth model (Appendix A) does not incorporate any effect of void spacing anisotropy, $\lambda$, in accord with void cell computations which showed very little effect of $\lambda$ as long as it lies in the range $1 / 2$ to 2 . Therefore, the effect of $\lambda$ on $J_{\text {Ic }} / \sigma_{0} X_{0}$ is due to the effect of the void spacing anisotropy on the coalescence mechanism. Another reason for the marked effect at high porosity comes from the definition of the void spacing $X_{0}$ as the distance from void center to void center. At some point,

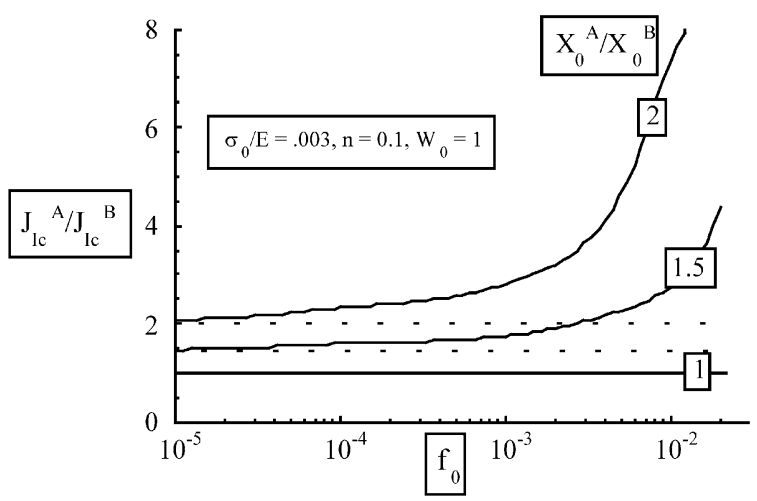

Fig. 11. Effect of the anisotropic void spacing on the ratio of toughness corresponding to two crack planes oriented at $90^{\circ}$ to one another. 
voids will touch each other in Specimen B for increasing porosity leading to zero toughness.

When the voids are non-spherical, void shape and void spacing effects are coupled. Two cases can be noted:

- Case 1 . The forming process usually elongates the second phases and increases the spacing in the same direction. Thus, Specimen B with $\lambda_{0}^{\mathrm{B}}>1$ would be expected to have voids with an initial prolate shape with respect to the loading direction. While Specimen A would have voids whose initial shape in the crack plane would be elliptical with greater cross-sectional area than the sphere with the same volume. Therefore, compared to the predictions based on initially spherical voids, Specimen B would have higher toughness and Specimen A would have lower toughness. The toughness ratio, $J_{\text {Ic }}^{\mathrm{A}} / J_{\text {Ic }}^{\mathrm{B}}=a$. could be either greater or smaller than unity depending on the tradeoff between the roles of spacing and initial void shape.

- Case 2. Specimen B with $\lambda_{0}^{\mathrm{B}}>1$ and with oblate void shapes relative to the loading direction. In this case, both the spacing and the initial void shape conspire to reduce the toughness for Specimen B relative to that of Specimen A.

Due to the complicated coupling between void aspect ratio and spacing effects, it is difficult to give general trends. Calculations based on an enhanced void growth model are essential. These combined effects probably explain why various degrees of fracture toughness anisotropy are observed in industrial alloys. To make meaningful predictions for a given material, it is essential to perform an in depth characterization of its microstructure. The purpose of Section 5 is to illustrate this point as well as to assess the present analysis for a specific set of experimental data.

\subsection{Physical versus engineering fracture toughness}

The engineering measurement of $J_{\text {Ic }}$ adopts the convention that initiation is associated with typically $0.2 \mathrm{~mm}$ of crack growth, corresponding to multiple void spacings for materials with closely spaced voids. The distinction between the 'first coalescence' estimate of initiation toughness invoked in the present paper and $J_{\text {Ic }}$ defined on the basis of $0.2 \mathrm{~mm}$ crack advance is illustrated in Fig. 12 in the case of two materials characterized by two different initial porosity, $f_{0}=10^{-3}$ and $f_{0}=$ $10^{-4}$. For a material exhibiting a void spacing of about $0.2 \mathrm{~mm}$, the engineering and physical toughness will not be much different as, on average, the initiation will involve a void located at $0.2 \mathrm{~mm}$ from the crack tip. However, if the void spacing is much smaller, for instance $0.02 \mathrm{~mm}$ as shown on Fig. 12, then the discrepancy between the physical initiation and engineering definition will be important, especially for low initial porosity. The difference between the two definitions is usually not an issue for structural integrity assessment since that is based on a full $R$-curve analysis, not just crack initiation. However, the difference can be important when comparing the toughness of different materials. The difference between the two definitions helps explaining results in the literature where the engineering fracture toughness appears abnormally large relative to what would be expected from (1) for materials with small void spacing $X_{0}$. For instance, in a remarkable set of experiments on the fracture toughness of high strength steels, Garrison and coworkers $[44,45]$ report several examples of inclusion spacings

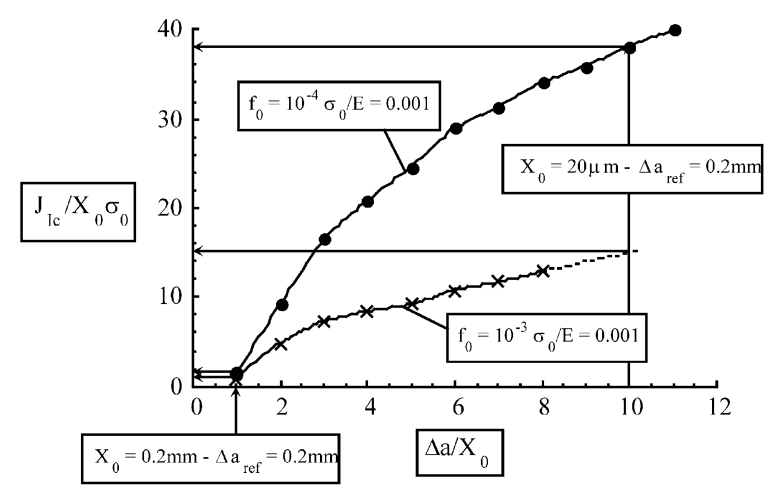

Fig. 12. Crack extension curves of two materials characterized by two different initial porosity $f_{0}=10^{-3}$ and $f_{0}=10^{-4}$. Effect of the initial void spacing on the engineering fracture toughness. 
between 1 and $10 \mu \mathrm{m} \quad\left(\sigma_{0} / E \approx 0.001\right.$ and $f_{0} \approx 10^{-4}$ ). Based on an engineering criterion, these materials exhibit critical crack tip opening displacements that range from 1 to 2 orders of magnitude larger than expected on the basis of the first coalescence criterion in Fig. 5 (i.e. $J_{\text {Ic }} / \sigma_{0} X_{0}=10$ to 100 ). The distinction between the two criteria highlights the arbitrariness of the concept of initiation toughness and importance of the $R$-curve, especially for very tough materials with high tearing resistance combined with small void spacings.

\section{Application to the fracture toughness anisotropy of steel}

The results described in Section 4 can be used to analyze the anisotropy in fracture toughness observed in materials with a preferred orientation of non-spherical second phases as well as second phase distributions with unequal spacings. As discussed in the last section, void shape and void distribution effects tend to be coupled in a way that depends significantly on the porosity. Well-defined toughness experiments can only be assessed by the model when reasonably detailed microstructural information is available. Some experimental investigations that report the necessary microstructural information are available in the literature, e.g. Refs. [10,11,21,27,33,40,46-54], but they are relatively scarce.

Bauvineau [33] conducted an investigation of the ductile fracture of rolled C-Mn steel, measuring the following fracture toughness for the directions ' $\mathrm{SL}$ ' and 'TL': $J_{\mathrm{Ic}}^{\mathrm{SL}} \approx 50 \mathrm{~kJ} / \mathrm{m}^{2}$ and $J_{\mathrm{Ic}}^{\mathrm{TL}} \approx 100 \mathrm{~kJ} / \mathrm{m}^{2}$ using the $J_{R}$ curve method based on $0.2 \mathrm{~mm}$ of crack growth. Here, L is the longitudinal direction, $\mathrm{T}$ is the transverse direction and $\mathrm{S}$ is the short transverse direction. SL means that the crack is in a plane orthogonal to the $\mathrm{S}$ direction and propagates in the L direction, while TL means that the crack is in a plane orthogonal to the $\mathrm{T}$ direction, propagating in the $\mathrm{L}$ direction.

Bauvineau also performed a detailed metallographic analysis of the inclusion population, which was primarily $\mathrm{MnS}$ inclusions. The second phase volume fraction was found equal to 0.0025 . This volume fraction can be considered as the initial void volume fraction, $f_{0}$, because void nucleation at the particles occurs readily. The inclusions are flat and elongated, presenting a preferential orientation due to the rolling process and display an ellipsoidal shape with mean diameters equal to 28 , 15 , and $3 \mu \mathrm{m}$ in the $\mathrm{L}, \mathrm{T}$ and $\mathrm{S}$ directions, respectively. For the purpose of applying our axisymmetric model to this material, the voids must be approximated by spheroids, i.e. ellipsoids with a circular projection in the equatorial plane. The effective diameter in the equatorial plane is chosen such that it gives the same projected area. The effective representative cell for the material strained in the $\mathrm{S}$ direction contains an oblate spheroid with effective diameters in the $\mathrm{L}$ and $\mathrm{T}$ directions $2 R_{0}=2.5 \mu \mathrm{m}$ and $2 R_{\mathrm{z} 0}=3 \mu \mathrm{m}$ respectively, i.e. $W_{0} \approx 1 / 7$. The representative cell for the $\mathrm{TL}$ tests is a more crude approximation of reality. The voids are approximated by prolate spheroid with an effective diameter $2 R_{0}=9.2 \mu \mathrm{m}$ while $2 R_{\mathrm{z} 0}=$ $15 \mu \mathrm{m}$, i.e. $W_{0} \approx 3 / 2$. To fully characterize the void cells, one needs a value for the void spacing in the $\mathrm{L}$ direction and a value for $\lambda_{0}$. In both SL and TL tests, the crack propagates in the $\mathrm{L}$ direction. Bauvineau has provided detailed measurements of the inclusion distribution using Voronoi cells and Delaunay triangulation methods. The mean void spacing between nearest neighbors in the $\mathrm{L}$ direction is equal to about $100 \mu \mathrm{m}$. (It is not obvious that the mean spacing is the most pertinent void spacing measure for present purposes; the average distance between nearest neighbors is another possibility.) The distance in the two other directions $\mathrm{T}$ and $\mathrm{S}$ are smaller than $100 \mu \mathrm{m}$ and not the same [53]. As it is difficult to have a reliable estimate of these two values, we have chosen, for simplicity to take $\lambda_{0} \approx 1$. This last assumption is reasonable in this case because the crack propagates in the $\mathrm{L}$ direction for both specimens and the respective spacings perpendicular to the plane of the crack are not very different.

The uniaxial flow properties are given by $\sigma_{0}=$ $290 \mathrm{MPa}$ and $n=0.25$. Plastic anisotropy is not significant. Table 1 summarizes the parameters characterizing the material when loaded in the SL and TL orientation and the predicted toughness. The 
Table 1

Microstructural parameters

\begin{tabular}{|c|c|c|c|c|c|c|}
\hline Direction & $f_{0}$ & $W_{0}$ & $\begin{array}{l}X_{0} \\
(\mu \mathrm{m})\end{array}$ & $\lambda_{0}$ & $\begin{array}{l}J_{\text {Ic }} \\
\text { exper. } \\
\left(\mathrm{kJ} / \mathrm{m}^{2}\right)\end{array}$ & $\begin{array}{l}J_{\text {Ic }} \\
\text { model } \\
\left(\mathrm{kJ} / \mathrm{m}^{2}\right)\end{array}$ \\
\hline SL & 0.0025 & $\begin{array}{c}3 / 22= \\
0.13\end{array}$ & 100 & 1 & 50 & 66 \\
\hline $\mathrm{TL}$ & 0.0025 & $\begin{array}{c}15 / 9= \\
1.7\end{array}$ & 100 & 1 & 100 & 145 \\
\hline
\end{tabular}

$f_{0}$ is the initial porosity, $W_{0}$ the initial void shape, $X_{0}$ is the void spacing and $\lambda_{0}$ is the initial void distribution parameter), measured and predicted fracture toughness for a C-Mn steel in the $\mathrm{SL}$ and TL directions. The steel is also characterized by $\sigma_{0}=$ $290 \mathrm{MPa}$ and $n=0.25$ with negligible plastic anisotropy.

difference in toughness between the two directions of propagation is well captured, and predicted and experimental are in reasonable agreement given the uncertainty in identifying the void spacing.

\section{Conclusions}

Small scale yielding finite element simulations of $2 \mathrm{D}$ crack growth in ductile materials represented by an enhanced void growth and coalescence model have been performed to elucidate the relation between fracture toughness, microstructure and flow properties. The main findings of the paper are:

- A distinct transition from a multiple void coalescence mechanism of crack growth to a void by void mechanism depending on initial porosity is confirmed [9].

- Proper modeling of void coalescence and accounting for the final rupture of the intervoid ligament is necessary to correctly capture the effect of microstructure changes on the fracture toughness. More experimental and theoretical work is required to better account for damage phenomena leading to final ligament fracture, such as the growth of a second population of voids [55] or microcleavage.

- The fracture toughness (normalized by the yield stress and void spacing) significantly decreases with decreasing hardening exponent. At least in some circumstances, this effect can explain why materials show lower toughness when their yield stress is elevated. If the strain-hardening remained unaltered, the toughness should increase. However, an increase in yield stress is frequently accompanied by a decrease in hardening, such that the net effect is a lowering of toughness.

- Void shape effects can justify the change of the fracture toughness with the orientation of loading axis and crack plane without invoking any plastic flow anisotropy.

- An anisotropic distribution of voids also significantly affects fracture toughness. This effect has received relatively little attention.

- The arbitrariness of the concept of initiation toughness and the importance of the $R$-curve, especially for very tough materials with high tearing resistance and small particle spacings has been highlighted. A single measure of fracture toughness for materials having low void spacing and exhibiting high tearing resistance is not likely to provide good index for comparing different materials.

Although the model used in the present paper is quite sophisticated, nevertheless, it does not rest on a truly realistic description of the microstructure of industrial metal alloys. Such alloys usually involve: (i) inhomogeneous void distribution (second phase clustering); (ii) a second population of voids; (iii) microscale gradients of properties (e.g. different phases, grain boundaries); (iv) competition with other failure modes [56]. Void nucleation may also significantly impact the trends described in this report, although it has been shown that the resistance to void nucleation should be high in order to affect the fracture toughness $[14,15]$. Considering all these complexities, it is reasonable to expect that the fracture toughness will continue to remain a phenomenological parameter to be determined experimentally. Trends such as those presented here serve mainly as a guide to ways in which fracture toughness can be improved via alterations in microstructure. 


\section{Acknowledgements}

The work of JWH was supported in part by NSF grant CMS-96-34632, by the AIM project through General Electric Company, and by the Division of Engineering and Applied Sciences, Harvard University.

\section{Appendix A. Enhanced model for void growth and coalescence}

\section{Void growth}

The void growth model is an extension of the Gurson [29] model proposed by Gologanu et al. [31], which has been modified to account for strain-hardening [30]. It is a full constitutive model for a porous elastoplastic material containing axisymmetric spheroidal voids. The parameters characterizing a representative volume element are defined on Fig. 1. The model, extended for strainhardening, contains nine state variables: the components of the mesoscopic stress tensor, $\Sigma_{i j}$, the porosity, $f$, the void aspect ratio, $S$, and an average yield stress for the matrix material, $\sigma_{\mathrm{y}}$. The void aspect ratio is defined by $S=\ln (W)$ where $W=R_{z} / R_{x}$. The equations of the model are

$$
\begin{aligned}
& \Phi_{\text {growth }} \equiv \frac{C}{\sigma_{\mathrm{y}}^{2}}\left\|\Sigma^{\prime}+\eta \Sigma_{\mathrm{h}} X\right\|^{2}+2 q(g \\
& \quad+1)(g+f) \cosh \left(\kappa \frac{\Sigma_{\mathrm{h}}}{\sigma_{\mathrm{y}}}\right)-(g+1)^{2}-q^{2}(g \\
& \quad+f)^{2}=0, \\
& \dot{f}=(1-f) \dot{E}_{k k}^{\mathrm{p}}, \\
& \dot{S}=\frac{3}{2}\left(1+h_{1}\right) X_{z i}\left(\dot{E}_{i j}^{\mathrm{p}}-\frac{\dot{E}_{k k}^{\mathrm{p}}}{3} \delta_{i j}\right) X_{\mathrm{z} j}+h_{2} \dot{E}_{k k}^{\mathrm{p}}, \\
& \sigma_{\mathrm{y}} \dot{\varepsilon}_{y}^{\mathrm{p}}(1-f)=\Sigma_{\mathrm{ij}} \dot{E}_{i j}^{\mathrm{p}}, \\
& \dot{E}_{i j}^{\mathrm{p}}=\gamma \frac{\partial \Phi}{\partial \Sigma_{i j}}
\end{aligned}
$$

where $\Phi$ is the flow potential, $\|$ is the von Mises norm, $\boldsymbol{\Sigma}^{\prime}$ is the deviatoric stress tensor, $\boldsymbol{\Sigma}_{\mathrm{h}}$ is the generalized hydrostatic stress defined by $\Sigma_{\mathrm{h}}=$ $\alpha_{2}\left(\Sigma_{x}+\Sigma_{y}\right)+\left(1-\alpha_{2}\right) \Sigma_{z}, \boldsymbol{X}$ is a tensor defined by $2 / 3 e_{z} \otimes e_{z}-1 / 3 e_{x} \otimes e_{x}-1 / 3 e_{y} \otimes e_{y}\left(\left(e_{x}, e_{y}, e_{z}\right)\right.$ is an orthogonal basis with $\boldsymbol{e}_{z}$ parallel to the cavity axis), $E^{\mathrm{p}}$ is the plastic strain tensor; (A2) and (A3) are the evolution laws for $f$ and $S$, respectively; (A4) is the Gurson [29] energy balance for the plastic work and allows computation of $\sigma_{\mathrm{y}}$ once the stress-strain curve for the matrix material is given; (A5) is the normality rule. The parameter $g$ is tied to the oblateness of the void and taken to be 0 for prolate and spherical voids. From their analysis, Gologanu et al. [31] derived analytical relationships for $C, \eta, g, \kappa, h_{1}, \alpha_{2}$ which can be expressed exclusively in terms of the state variables $S$ and $f$, except for $h_{1}$ which also depends on the stress triaxiality. The heuristic parameter $q$ depends on $n, f_{0}$, and $S$ in order to obtain accurate predictions towards void cells calculations (see [30] for the details about the expression of the dummy variables).

\section{Void coalescence}

The coalescence model, which derives from Thomason's criterion for the onset of coalescence [32], directly addresses the mechanism of tensile plastic localization in the intervoid ligaments. In Ref. [30], the criterion of Thomason has been extended to the complete coalescence process and also to strain-hardening materials. In order to couple the coalescence model with the void growth model (A1)-(A5), a new state variable related to the void distribution, $\chi=L_{x} / R_{x}$ has been introduced. The coalescence model was elaborated based on the observation that void coalescence corresponds to a localization of plasticity in the void ligament and a transition to a uniaxial mode of loading. Evolution law for the geometrical variables $(W, f$ and $\chi$ ) as well as for the axial stress and yield stress have been given based on the approximation that the voids remain ellipsoidal. For the sake of implementing the model in a finite element setting, we found more adequate to rewrite the coalescence model within the framework of a classical plasticity model assuming a normality rule [34]. The void coalescence model writes:

$$
\Phi_{\text {coalescence }} \equiv \frac{\Sigma_{\mathrm{e}}}{\sigma_{\mathrm{y}}}+\frac{3}{2} \frac{\left|\Sigma_{\mathrm{h}}\right|}{\sigma_{\mathrm{y}}}-F(W, \chi)=0
$$


where

$$
\begin{aligned}
& F(W, \chi) \equiv \frac{3}{2}\left(1-\chi^{2}\right)\left[\alpha\left(\frac{1-\chi}{\chi W^{2}}\right)^{2}+\beta \sqrt{\frac{1}{\chi}}\right] \\
& \dot{W}=\frac{3 W\left(2 \chi^{2}-1\right)}{4 f} \dot{E}_{\mathrm{e}}^{\mathrm{p}}, \\
& \dot{\chi}=\frac{\chi\left(3-2 \chi^{2}\right)}{4 f} \dot{E}_{\mathrm{e}}^{\mathrm{p}} \\
& \dot{f}=(1-f) \dot{E}_{k k}^{\mathrm{p}}, \\
& \dot{\sigma}_{\mathrm{y}}=\frac{\partial \sigma_{\mathrm{y}}}{\partial \varepsilon_{\mathrm{y}}^{\mathrm{p}}} \frac{\chi^{2}}{f} \dot{E}_{\mathrm{e}}^{\mathrm{p}}, \\
& \dot{E}_{i j}^{\mathrm{p}}=\gamma \frac{\partial \Phi}{\partial \Sigma_{i j}} .
\end{aligned}
$$

The parameter $\alpha$ is given by the fit (for $0 \leq n \leq$ 0.3) $\alpha=0.1+0.217 n+4.83 n^{2}$, while $\beta$ can be considered as constant and equal to 1.24.

\section{References}

[1] Hutchinson JW. J Appl Mech 1983;50:1042.

[2] Aravas N, McMeeking RM. J Mech Phys Solids 1985;33:25.

[3] Needleman A, Tvergaard V. J Mech Phys Solids 1987;35:151.

[4] Hom CL, McMeeking RM. J Mech Phys Solids 1989;37:395.

[5] Tvergaard V, Needleman A. J Mech Phys Solids 1992;40:447.

[6] Ghosal AK, Narasimhan R. Int J Fract 1996;77:281.

[7] Gao X, Shih CF, Tvergaard V, Needleman A. J Mech Phys Solids 1996;44:1255.

[8] Yan C, Mai Y-W. Int J Fract 1998;92:287.

[9] Tvergaard V, Hutchinson J.W. Mech report 372. Harvard University; 2001.

[10] Mudry F, di Rienzo F, Pineau A. In: Landes JD, Saxena A, Merkle JG, editors. Non-linear fracture mechanics: Volume II-elastic-plastic fracture. ASTM STP 995. Philadelphia: ASTM; 1989. p. 24.

[11] Rousselier G, Devaux J-C, Mottet G, Devesa G. In: Landes JD, Saxena A, Merkle JG, editors. Nonlinear fracture mechanics: Volume II-elastic-plastic fracture. ASTM STP 995. Philadelphia: ASTM; 1989. p. 332.

[12] Bilby BA, Howard IC, Li ZH. Fatigue Fract Eng Mater Struct 1993;16:1.

[13] Xia L, Shih CF, Hutchinson JW. J Mech Phys Solids 1995;43:389.

[14] Xia L, Shih CF. J Mech Phys Solids 1995;43:233.

[15] Xia L, Shih CF. J Mech Phys Solids 1995;43:260.
[16] Brocks W, Klingbeil D, Kunecke G, Sun D-Z. In: Kirk M, Bakker A, editors. Constraint effects in fracture theory and applications. ASTM STP 1244, vol. 2. Philadelphia: ASTM; 1995. p. 232.

[17] Ruggieri C, Panontin TL, Dodds Jr. RH. Int J Fract 1996;82:67.

[18] Gao X, Faleskog J, Dodds RH, Shih CF. Eng Fract Mech 1998;59:761.

[19] Siegmund T, Brocks W. Int J Fract 1999;99:97.

[20] Zhang ZL, Thaulow C, Odegard J. Eng Fract Mech 2000;67:155.

[21] Rivalin F, Besson J, Pineau A, Di Fant M. Eng Fract Mech 2001;68:347.

[22] Tvergaard V, Hutchinson JW. J Mech Phys Solids 1992;40:1377.

[23] Tvergaard V, Hutchinson JW. Int J Solids Struct 1996;33:3297.

[24] Siegmund T, Brocks W. Eng Fract Mech 2000;67:139.

[25] Roy YA, Dodds RH. Int J Fract 2001;110:21.

[26] Rice JR, Johnson MA. In: Kanninen MF, Adler WF, Rosenfield AR, Jaffee RI, editors. Inelastic behavior of solids. McGraw-Hill; 1970. p. 641.

[27] Mudry F. Etude de la Rupture Ductile et de la Rupture par Clivage d'Aciers Faiblement Alliés. PhD thesis (Thèse d'état) Université de Technologie de Compiègne, France; 1982.

[28] Ritchie RO, Thompson AW. Metall Trans A 1985;16:233.

[29] Gurson AL. J Eng Mater Tech 1977;99:2.

[30] Pardoen T, Hutchinson JW. J Mech Phys Solids 2000;48:2467.

[31] Gologanu M, Leblond J-B, Perrin G, Devaux J. In: Suquet $\mathrm{P}$, editor. Continuum micromechanics. Berlin: SpringerVerlag; 1995. p. 61.

[32] Thomason PF. Ductile fracture of metals. Oxford: Pergamon Press, 1990.

[33] Bauvineau L. Approche Locale de la Rupture Ductile: Application à un Acier Carbone-Manganèse. $\mathrm{PhD}$ thesis. Ecole Nationale Supérieure des Mines de Paris, France; 1996.

[34] Benzerga AA. J Mech Phys Solids 2002;50:1331.

[35] ABAQUS 5.8. User's manual, Hibbit, Karlsson and Sorensen, providence. R.I., US; 1997.

[36] Simo JC, Hughes TJR. Computational inelasticity. NewYork: Springer-Verlag, 1998.

[37] Aravas N. Int J Numer Meth Eng 1987;24:1395.

[38] Tvergaard V. Adv Appl Mech 1990;27:83.

[39] Knott JF. Metal Sci 1980;:327.

[40] Pardoen T. Ductile fracture of cold-drawn copper bars: experimental investigation and micromechanical modeling. $\mathrm{Ph} \mathrm{D}$ thesis. Université catholique de Louvain, Belgium; 1998.

[41] Luo LG, Ryks A, Embury JD. Metallography 1989;23:101.

[42] Dumont D, Deschamps A, Brechet Y. Acta mater (Submitted).

[43] Dumont D. Relations microstructure/ténacité dans les alliages aéronautiques de la série 7000 . $\mathrm{PhD}$ thesis. Institut National Polytechnique de Grenoble, France; 2001. 
[44] Garrison Jr. WM, Wojcieszynski AL, Iorio LE. In: Mahidhara RK, Geltmacher AB, Matic P, Sadananda K, editors. Recent advances in fracture. TMS; 1997. p. 155.

[45] Bray JW, Maloney JL, Raghavan KS, Garrison WM. Metall Trans A 1991;22:2277.

[46] Bourcier RJ, Koss DA, Smelser RE, Richmond O. Acta Metall 1986;34:2443.

[47] Becker R. J Mech Phys Solids 1987;35:577.

[48] Becker R, Smelser RE, Richmond O. J Mech Phys Solids 1989;37:111.

[49] Steglish D, Siegmund T, Brocks W. Comput Mater Sci 1999;16:404.

[50] Decamp K, Bauvineau L, Pineau A. Int J Fract 1997;88:1.
[51] Pardoen T, Doghri I, Delannay F. Acta mater 1998;46:541.

[52] Benzerga AA, Besson J, Pineau A. J Eng Mater Tech 1999;121:221.

[53] Benzerga AA. Rupture ductile de tôles anisotropes. PhD thesis. Ecole Nationale Supérieure des Mines de Paris; 2000.

[54] Besson J, Devillers-Guerville L, Pineau A. Eng Fract Mech 2000;67:169.

[55] Faleskog J, Shih CF. J Mech Phys Solids 1997;45:21.

[56] Pineau A. In: Bakker A, editor. Mechanical behaviour of materials. Delft, The Netherlands: Delft University Press; 1995. p. 1. 